Revue d'histoire de l'Amérique française

Q8. REVUE D.HISTOIRE DE L'AMÉRIQUE FRANÇAISE

\title{
Une bureaucratie en voie de formation
}

\section{L'administration municipale de Montréal dans la première moitié du $\mathrm{XX}^{\mathrm{e}}$ siècle}

\section{Michèle Dagenais}

Volume 46, numéro 1, été 1992

Montréal 1642-1992

URI : https://id.erudit.org/iderudit/305052ar

DOI : https://doi.org/10.7202/305052ar

Aller au sommaire du numéro

Éditeur(s)

Institut d'histoire de l'Amérique française

ISSN

0035-2357 (imprimé)

1492-1383 (numérique)

Découvrir la revue

Citer cet article

Dagenais, M. (1992). Une bureaucratie en voie de formation : l'administration municipale de Montréal dans la première moitié du $\mathrm{XX}^{\mathrm{e}}$ siècle. Revue d'histoire de l'Amérique française, 46(1), 177-205. https://doi.org/10.7202/305052ar
Résumé de l'article

Par un retour sur les étapes charnières de l'histoire politique de Montréal, cet article vise à retracer certaines des dynamiques à l'oeuvre dans le processus de bureaucratisation de l'administration municipale au cours de la première moitié du XX ${ }^{\mathrm{e}}$ siècle. Si le projet de transformation de l'appareil administratif émerge d'abord dans le contexte des luttes qui opposent les populistes aux réformistes, il est rapidement repris par d'autres acteurs présents à l'Hôtel de Ville. C'est pourquoi, au-delà des luttes électorales, il importe aussi d'analyser les rivalités entre hommes politiques et fonctionnaires, pour saisir dans toute sa complexité le processus de formation de la bureaucratie municipale.
Tous droits réservés @ Institut d'histoire de l'Amérique française, 1992
Ce document est protégé par la loi sur le droit d'auteur. L'utilisation des services d’Érudit (y compris la reproduction) est assujettie à sa politique d'utilisation que vous pouvez consulter en ligne.

https://apropos.erudit.org/fr/usagers/politique-dutilisation/ 


\title{
UNE BUREAUCRATIE EN VOIE DE FORMATION \\ L'ADMINISTRATION MUNICIPALE DE MONTRÉAL DANS LA PREMIÈRE MOITIÉ DU XX ${ }^{\mathrm{e}}$ SIÈCLE $^{1}$
}

\author{
MICHÈLE DAGENAIS \\ Département d' histoire \\ Université du Québec à Montréal
}

\section{RÉSUMÉ}

Par un retour sur les étapes charnières de l'histoire politique de Montréal, cet article vise à retracer certaines des dynamiques à l'œuvre dans le processus de bureaucratisation de l'administration municipale au cours de la première moitié du $\mathrm{XX}^{\mathrm{e}}$ siècle. Si le projet de transformation de l'appareil administratif émerge d'abord dans le contexte des luttes qui opposent les populistes aux réformistes, il est rapidement repris par d'autres acteurs présents à l'Hôtel de Ville. C'est pourquoi, audelà des luttes électorales, il importe aussi d'analyser les rivalités entre hommes politiques et fonctionnaires, pour saisir dans toute sa complexité le processus de formation de la bureaucratie municipale.

\section{ABSTRACT}

Through a survey of the turning points of Montreal political history, this article aims to retrieve some dynamics of the city's administration bureaucratization, during the first half of the twentieth century. Transforming the administrative system is an idea that first arose in the context of the struggle between populists and reformists, but was soon taken up by other actors at City Hall. It is therefore important to analyse, beyond electoral struggles, the rivalries between politicians and civil servants, if we are to understand the complex forming process of that bureaucracy.

1 Cet article s'inscrit dans le cadre plus vaste de mes recherches de doctorat: Dynamiques d'une bureaucratie: l'administration municipale de Montréal et ses fonctionnaires, 1900-1945, thèse de Ph.D. (histoire), Université du Québec à Montréal, 1992. Cette recherche a bénéficié de l'appui financier du Conseil de recherches en sciences humaines du Canada. Je remercie aussi Lucia Ferretti, Jean-Pierre Collin, Paul-André Linteau et Bettina Bradbury de leurs commentaires. 
L'administration municipale est au cœur du processus d'urbanisation intense qui caractérise Montréal au tournant du $\mathrm{XX}^{\mathrm{e}}$ siècle. C'est au cours de cette période que sont graduellement mises en place de nombreuses infrastructures, et développés divers services pour faire face aux besoins grandissants de la Ville en pleine expansion. Parce qu'ainsi appelée à jouer un rôle de premier plan, l'administration montréalaise est l'objet de nombreuses luttes politiques locales. Sur la scène électorale, populistes et réformistes s'opposent en vue d'occuper les postes à l'Hôtel de Ville.

L'Hôtel de Ville est alors sous la coupe de politiciens accusés de corruption et de manœuvres frauduleuses. Détenant une bonne partie des sièges au Conseil municipal, ils se retrouvent aux principaux postes de commande, ceux des commissions échevinales chargées de l'administration des différents services: voirie, aqueduc, santé, sécurité publique, incendie. Par la distribution de généreux contrats et la création d'emplois, les politiciens se constituent des clientèles politiques et s'assurent la fidélité d'une partie importante de l'électorat.

Le mouvement réformiste dénonce vertement ces pratiques de patronage. À partir de la fin du XIX ${ }^{\mathrm{e}}$ siècle, ses membres, principalement des hommes d'affaires, tentent à leur tour d'obtenir la direction de l'Hôtel de Ville avec l'objectif bien arrêté de dépolitiser l'administration. Ils espèrent ainsi soustraire la gestion des affaires municipales aux influences politiques, en la confiant à des experts et à des administrateurs plus susceptibles, à leur avis, de diriger efficacement la Ville. La mise en œuvre de ce programme, qui vise à moyen terme l'assainissement et la rationalisation de l'administration municipale, passe par une redistribution des pouvoirs au profit de gestionnaires non élus.

Cette lutte de pouvoir entre populistes et réformistes sur la scène électorale se double de la rivalité plus fondamentale entre politiciens et administrateurs sur le partage des pouvoirs au sein de l'administration et sur la manière de gérer les affaires publiques. Or cette dimension de l'histoire des gouvernements est beaucoup moins connue car l'éclairage a davantage été dirigé vers les faits et gestes des hommes politiques, laissant ainsi dans l'ombre le rôle actif des administrateurs et des techniciens dans le développement d'une bureaucratie permanente ${ }^{2}$.

2 En histoire urbaine, une des rares études à s'être penchées sur cette question est celle de Jon C. Teaford, The Unheralded Triumph. City Government in America, 1870-1900 (Baltimore, John Hopkins University Press, 1984), 365 p. Voir le bilan de Raymond A. Mohl, «New Perspectives on American Urban History», The Making of Urban America (Wilmington, Del., Scholarly Resources, 1988), 203-316. 
Ces luttes et ces rivalités entre hommes politiques et bureaucrates constituent le creuset dans lequel prend naissance le processus de bureaucratisation de l'administration municipale montréalaise. Si bien d'autres facteurs y contribuent à leur tour, c'est d'abord à cette dimension que notre article est consacré. Par un retour sur les étapes charnières de l'histoire politique de Montréal, nous tenterons de retracer comment les premières volontés des réformistes prennent graduellement forme et sont ensuite reprises par d'autres acteurs également présents à l'Hôtel de Ville. L'accent sera donc mis sur les étapes les plus visibles de ce processus au cours duquel l'administration montréalaise se transforme radicalement et adopte la forme d'une bureaucratie «moderne».

La bureaucratie est ici comprise comme une forme d'organisation spécifique, mue par une dynamique de pouvoir ${ }^{3}$. Ce type d'organisation se caractérise par certains traits généraux dont les plus connus sont: la présence d'un ensemble de règles et de règlements écrits qui définissent tous les aspects de son fonctionnement; l'existence d'une organisation du travail basée sur une division hiérachique des tâches; la concentration du pouvoir dans les échelons supérieurs de cette hiérarchie. Bien que cette définition sommaire se réfère explicitement au modèle webérien, à son idéal-type ${ }^{4}$, il ne saurait cependant être question de se limiter à cette vision formaliste, statique. En effet, la bureaucratie est un phénomène bien antérieur, dans le temps, à cette définition formelle. Plus qu'un processus interne de développement des organisations, la bureaucratie est d'abord une forme d'organisation sociale qui se retrouve à diverses époques et en de multiples endroits. La difficulté de définir ce concept réside ainsi dans la multiplicité de sens qu'on lui accorde. Pour nous, le concept de bureaucratie recouvre une double dynamique: il s'agit à la fois d'un mode d'organisation, ou plus précisément d'un mode de domination sociale, et d'un processus qui se généralise et se perpétue. Cette définition suppose donc qu'on s'interroge non seulement sur les formes prises par ce type d'organisation mais aussi sur sa genèse, sur les étapes suivant lesquelles il se développe et s'élabore.

Cet article vise à retracer ce processus en identifiant certaines des dynamiques à l'origine de la transformation de l'appareil administratif municipal. On analysera les difficultés que pose la transformation de

3 Cette réflexion sur la bureaucratie s'inspire principalement des travaux de Georges Lapassade, Groupes, organisations et institutions (Paris, Gauthier-Villars Éditeur, 1970); et de Claude Lefort, «Qu'est-ce que la bureaucratie?», Éléments d' une critique de la bureaucratie (Paris, Gallimard, 1979), 271-301.

4 Max Weber, Économie et société (Paris, Plon, 1971), 219-252. 
cette institution, les nombreux obstacles qui s'opposent au projet, d'abord formulé par les réformistes du début du siècle, de la doter d'un type d'organisation moderne et bureaucratique. Si, pour les uns, cette difficulté est imputable aux réformistes eux-mêmes et à leur incapacité à s'imposer sur la scène politique ${ }^{5}$, ou encore à la culture politique des francophones ${ }^{6}$, pour d'autres, elle trouve son explication dans les caractéristiques mêmes de la vie politique municipale ${ }^{7}$. Selon le politicologue Guy Bourassa, la principale résistance vis-à-vis des réformes provient des élus locaux eux-mêmes, dans la mesure où la mise en place de structures bureaucratiques qu'elles induisent a pour effet de leur enlever certains de leurs pouvoirs et de leurs attributions. C'est pourquoi, constate-t-il, les principales réformes de l'appareil administratif ont lieu durant les périodes de tutelle, au moment où le Conseil municipal est affaibli et ne jouit pas de ses pleins pouvoirs.

Si cette conception de Guy Bourassa s'avère en partie juste, elle n'en demeure pas moins réductrice de la complexité des dynamiques à l'œuvre dans le processus de bureaucratisation de l'administration municipale. Bien que les périodes de mise en tutelle se caractérisent par des changements plus marquants, plusieurs réformes, comme nous le verrons, sont aussi adoptées à des époques où les élus municipaux jouissent de leurs pleins pouvoirs. Surtout, la consolidation des changements se produit bien souvent à d'autres moments, sous l'effet de l'action d'autres groupes présents à l'Hôtel de Ville. Des dynamiques plus internes doivent donc également être considérées pour comprendre à la fois les facteurs qui favorisent ce processus de bureaucratisation et les forces d'inertie qui tendent, au contraire, à le ralentir.

L'administration municipale, c'est tout un monde en soi, composé des élus, des bureaucrates, de même que de milliers de fonctionnaires. $\mathrm{Si}$ certains y interviennent de manière plus visible et plus spectaculaire, c'est aussi par un processus quotidien que cet univers se façonne au fil des ans. Tous ne possèdent évidemment pas le même

5 C'est notamment la thèse défendue par Michael Gauvin, «The Reformer and the Machine: Montreal Civic Politics from Raymond Préfontaine to Médéric Martin», Journal of Canadian Studies/Revue d'études canadiennes, 13,2 (été 1978): 16-26, et Francine Nagant, La politique municipale a Montréal, de 1910 a 1914: l'échec des réformistes et le triomphe de Médéric Martin, mémoire de M.A. (histoire), Université de Montréal, 1983.

6 Harold Kaplan, Reform, Planning, and City Politics: Montreal, Winnipeg, Toronto (Toronto, University of Toronto Press, 1982). Cet auteur est sans doute un des représentants les plus connus de cette interprétation culturaliste.

7 C'est la thèse avancée par Guy Bourassa, Les relations ethniques dans la vie politique montréalaise, chapitre premier, "Le système politique montréalais», Commission royale d'enquête sur le bilinguisme et le biculturalisme, Ottawa, Informations Canada, 1971, 70. Cette thèse est reprise par Annick Germain, Les mouvements de réforme urbaine à Montréal, de 1880 d 1920, thèse de Ph.D. (sociologie), Université de Montréal, 1980, 234. 
pouvoir d'orienter son évolution, mais chacun à sa manière n'en contribue pas moins à modeler son fonctionnement. Tantôt les élus occupent le devant de la scène, tantôt les chefs de service, tantôt les fonctionnaires.

Notre article est divisé en deux grandes parties qui épousent le rythme des transformations de l'administration montréalaise durant la première moitié du $\mathrm{XX}^{\mathrm{e}}$ siècle. La première période, du début du siècle à la veille de la crise, s'apparente à une phase de lente élaboration marquée par l'adoption de plusieurs réformes. C'est à ce moment que se dessine le projet bureaucratique. Au cours de la seconde période, de 1930 à 1944, les événements s'accélèrent. Le contexte de crise économique, qui affecte durement l'administration municipale, insuffle une nouvelle dynamique au processus de bureaucratisation. On assiste à la consolidation de plusieurs des réformes entreprises précédemment. Si des mouvements extérieurs sont à l'origine des transformations, l'impulsion première au cours de cette seconde période, provient essentiellement des agents à l'œuvre au sein même de l'Hôtel de Ville.

\section{- I -}

\section{1 - L'ADMINISTRATION MUNICIPALE AU TOURNANT DU SIĖCLE ET LE MOUVEMENT DE RÉFORME URBAINE}

Quoiqu'en plein essor, l'administration municipale est encore peu développée au tournant du siècle ${ }^{8}$. L'Hôtel de Ville est alors géré essentiellement par les élus: le maire et une trentaine de conseillers qui représentent les différents quartiers de la Ville. À l'issue de chaque scrutin, le Conseil puise parmi les élus les membres des commissions chargées de l'administration des services municipaux: hygiène, police, incendie, chemins, éclairage, aqueduc. La commission des finances occupe une place prépondérante dans cet ensemble puisque c'est elle qui dresse les prévisions budgétaires annuelles. Elle possède également le droit de regard sur toute dépense et tout contrat non prévus dans le budget, qui doivent recevoir son approbation avant d'être soumis au vote du Conseil municipal ${ }^{9}$.

8 Pour une vue d'ensemble de l'administration municipale à cette époque: Paul-André Linteau, Histoire de Montréal depuis la Confédération (Montréal, Boréal, 1992), chapitres cinq et dix. Voir aussi J. Cléophas Lamothe, Histoire de la Corporation de la Cité de Montréal depuis son origine jusqu'd nos jours (Montréal, 1903), 65-197; Camille Bertrand, Histoire de Montréal, Tome 2: (1760-1942) (Montréal, 1942), chapitre huit; Honoré Parent, "L'administration municipale», Esdras Minville, dir., Montréal économique (Montréal, Fides/HEC, 1943), $327-$ 375.

9 Honoré Parent, loc. cit., 360; Charte de la Cité de Montréal, 62 Victoria, chap. 58, 1899 , article 42 . 
Ce sont ces commissions échevinales qui dirigent véritablement les destinées de la Ville, bien davantage que le Conseil et le maire ${ }^{10}$. Mais parce qu'elles sont formées uniquement d'élus, leur composition change souvent d'une élection à l'autre, ce qui compromet la continuité de l'administration. En outre, aucune instance formelle n'est chargée d'assurer la coordination des travaux des commissions, si bien que les empiétements entre les diverses juridictions sont forcément nombreux ${ }^{11}$.

De l'avis des réformistes, le principal problème de ce mode de fonctionnement, c'est qu'il favorise nettement les pratiques de favoritisme et de corruption. Les présidents des commissions, prétendentils, sont ainsi plus sensibles aux besoins des électeurs des quartiers qu'ils représentent qu'à ceux de la Ville dans son ensemble. La «tentation» de choyer leurs électeurs est particulièrement grande chez les membres des comités qui doivent faire appel à des entrepreneurs indépendants pour effectuer les nombreux travaux de construction de rues, d'installations sanitaires, etc. L'attribution de tous ces contrats représente autant d'occasions de distribuer concessions et emplois, et permet aux conseillers de se bâtir des clientèles politiques.

Les hommes d'affaires et les propriétaires, membres d'associations telles le Board of Trade, la Chambre de commerce ou la Real Estate Owners Association, constituent les principaux représentants du mouvement de réforme urbaine à Montréal ${ }^{12}$. C'est par eux que sont formulées les premières volontés de transformer l'administration montréalaise, de la «moderniser». Regroupés au sein du Reform Party en prévision des élections municipales de 1900, ils remportent la victoire. Tout comme leurs voisins du Sud dont ils s'inspirent, les réformistes au pouvoir vont d'abord prétendre éliminer le patronage, puis chercher à accroître l'efficacité de l'administration en vue

10 Camille Bertrand, op. cit., 142. Voir aussi l'ouvrage de John C. Weaver, Shaping the Canadian City: Essays on Urban Politics and Policy, 1890-1920 (L'Institut d'administration publique du Canada, 1977), et en particulier le chapitre quatre, «Municipal Reform: the Corporate Ideal and New Structures of Government», 56.

11 Selon J.-C. Lamothe, il existait alors un Bureau des présidents des commissions échevinales qui avait pour fonction de discuter de l'allocation des crédits. L'auteur ne donne cependant pas de détails sur le mode de fonctionnement de cette instance; op. cit., 77. Nous n'avons pas trouvé trace du Bureau dans les archives municipales.

12 Francine Nagant, op. cil., 17. Sur cette période bien connue de l'histoire politique de Montréal, citons également l'article de Michael Gauvin, «The Reformer and the Machine...», qui reprend les principaux résultats de son mémoire de maîtrise, The Municipal Reform Movement in Montreal, 1886-1914, mémoire de M.A. (histoire), Université d'Ottawa, 1972, 163 p. Mentionnons également les recherches de Annick Germain, op. cit., de même que celles de Daniel J. Russell, H.B. Ames as Municipal Reformer, mémoire de M.A. (histoire), McGill University, 1972, $169 \mathrm{p}$. 
d'assainir la gestion des affaires municipales et de maintenir l'imposition au niveau le plus bas possible ${ }^{13}$. Dans cette optique, les réformistes montréalais forment une commission spéciale chargée d'enquêter sur les méthodes d'embauche des fonctionnaires et l'organisation du travail ${ }^{14}$. La commission recommande, entre autres, que les candidats à la fonction publique municipale soient tenus de passer un examen. Elle propose aussi de réglementer les promotions suivant le mérite des fonctionnaires et d'établir une échelle de salaires qui fixerait les augmentations à l'intérieur de chaque échelon hiérarchique $^{15}$. Cependant, aucune mesure concrète n'est mise en œuvre avant la fin du mandat des réformistes. Leur première victoire est de courte durée puisqu'aux élections suivantes, en 1902, le nombre de leurs élus diminue nettement.

Avec les années le mouvement montréalais, qui s'inspire fortement de la réussite des expériences menées par les réformistes aux États-Unis, propose un programme plus cohérent et plus complet en vue d'améliorer la gestion des affaires municipales ${ }^{16}$. Il est également influencé en cela par l'idéologie du modern management et de la gestion scientifique du travail, développée à la même époque par W. F. Taylor et ses disciples ${ }^{17}$. Les réformistes s'orientent alors plus directement vers la réforme des structures. En vue de soustraire la gestion des affaires municipales à l'influence des intérêts particuliers, ils préconisent la tenue d'élections à la grandeur de la Ville plutôt qu'à l'échelle des quartiers, et la centralisation des décisions au sein d'un organisme exécutif.

Plus concrètement, la dépolitisation visée de l'administration nécessite une division des pouvoirs législatif, exécutif et administratif, jusque-là confondus et exercés uniquement par les élus. On espère ainsi séparer l'étape de la formulation d'objectifs d'ensemble de celle de leur réalisation. Si les réformistes concèdent que l'élaboration de projets appartient en propre à la sphère politique, ils estiment en

13 Pour une vue d'ensemble des mouvements de réforme urbaine aux États-Unis et pour un aperçu de l'historiographie américaine sur la question, voir l'étude de Raymond $\mathrm{A}$. Mohl, The New City. Urban America in the Industrial Age, 1860-1920 (Illinois, Harland Davidson Inc., The American History Series, 1985), 81-242.

14 Ville de Montréal. Division de la gestion de documents et des archives (DGDA), Commission des finances, rapport n ${ }^{\circ} 854,14$ janvier 1901; voir aussi ${ }^{\circ} 1329,10$ décembre 1900.

15 Tout comme les autres, cette dernière mesure ne se concrétisera pas, contrairement à ce que laisse entendre Michael Gauvin dans son mémoire, op. cit., 55.

16 Francine Nagant, op. cit., 26.

17 Sur la question des sources d'inspiration des réformistes et de l'influence des théories du modern management, voir l'étude de Samuel Haber, Efficiency and Uplift. Scientific Management in the Progressive Era 1890-1920 (Chicago, University of Chicago Press, 1964), chapitre six. 
revanche que leur concrétisation doit être confiée aux experts et aux administrateurs. La séparation de la fin et des moyens ainsi préconisée représente la meilleure garantie, à leur avis, que les problèmes seront traités de manière impartiale, objective et scientifique. Dans les faits, cette solution a pour but de soustraire un nombre croissant de décisions au processus démocratique, au profit de la sphère exécutive ou administrative. On peut dès lors facilement comprendre que l'opposition manifestée par les politiciens, et en particulier par les populistes, ne vise pas tant le projet de modernisation de l'administration municipale en soi, mais bien plutôt le risque évident de leur subordination aux administrateurs.

\section{2 - UNE PREMIÈRE RÉFORME DES INSTITUTIONS MUNICIPALES: LA NAISSANCE DU BUREAU DES COMMISSAIRES ${ }^{18}$}

Regroupés au sein du tout nouveau Comité des citoyens, les réformistes montréalais ${ }^{19}$ lancent une nouvelle offensive en 1908, dans le but de transformer en profondeur l'administration de la Ville. Le Comité fait de multiples pressions auprès du gouvernement provincial pour qu'il dote la Ville d'un Bureau des commissaires, à l'image des bureaux existant dans les autres municipalités canadiennes ou américaines. On suggère qu'il soit composé de quatre membres élus par l'ensemble de la Ville, et non plus par les citoyens de chaque quartier, cherchant ainsi à neutraliser l'influence jugée néfaste des électeurs sur les conseillers. Le gouvernement provincial, qui voit d'un mauvais œil la trop grande influence dont bénéficient les populistes, appuie le projet réformiste. Mais par crainte de soulever l'opposition de certains Montréalais, il décide de soumettre la réforme à la population par voie de référendum, plutôt que de l'imposer ${ }^{20}$. Du même souffle, le Comité des citoyens exige la tenue d'une commission d'enquête publique sur l'administration de Montréal, ce qu'il obtient également. Nommée en avril 1909, la commission ${ }^{21}$ remet au mois de décembre suivant un

18 Les contemporains emploient indistinctement les termes Bureau de contrôle ou Bureau des commissaires lorsqu'ils se réfèrent à cette institution. Le premier terme, toutefois, constitue la traduction littérale du vocable employé en anglais: «Board of Control». Comme dans les textes de loi municipaux se rapportant à l'institution montréalaise on emploie le terme Bureau des commissaires, c'est ce dernier que nous utiliserons. Se référer aux Amendements a la charte de la Cité de Montréal (Montréal, 1910).

19 Ce nouveau regroupement réformiste réunit sensiblement les mêmes personnes qu'au début du siècle, soit les gens proches du milieu des affaires et du commerce: Michael Gauvin, loc. cit., 20.

20 Robert Rumilly, Histoire de Montréal, tome 3, 1867-1918 (Montréal, Fides, 1972), 402 .

21 Commission royale d'enquête sur l'administration municipale de Montréal, présidée par le juge Lawrence John Cannon. 
rapport dénonçant fermement le patronage qui sévit à l'Hôtel de Ville ${ }^{22}$.

Entre-temps, les Montréalais sont appelés à se prononcer sur le nouveau mode de gouvernement municipal et votent majoritairement pour l'établissement d'un Bureau des commissaires. Aux élections de février 1910, les réformistes, à l'origine de tout ce remue-ménage, remportent facilement la victoire: ils obtiennent les quatre nouveaux postes de commissaires et plus de la moitié des 31 sièges de conseillers ${ }^{23}$.

L'avènement du Bureau des commissaires constitue une étape majeure dans la modernisation de l'administration municipale. Il représente une tentative de délimitation plus précise des pouvoirs formels, alors partagés entre le Conseil municipal et les commissions échevinales ${ }^{24}$. Désormais les fonctions exécutives et administratives sont confiées à la nouvelle institution, tandis que le Conseil conserve ses attributions dans le domaine législatif. Le Bureau hérite ainsi de tous les pouvoirs administratifs autrefois exercés par les commissions échevinales. Il se voit aussi confier l'importante tâche de préparer le budget et de le gérer, après approbation du Conseil. Le nouveau Bureau des commissaires est en outre responsable de la nomination et de la révocation de la majorité des fonctionnaires ${ }^{25}$.

Malgré les principes d'efficacité qui les animent, la gestion des réformistes au pouvoir souffre de plusieurs lacunes et se révèle rapidement inadéquate. Le Bureau des commissaires ne parvient manifestement pas à remplir son mandat et croule sous l'avalanche des responsabilités qui lui incombent. C'est que toute décision concernant le fonctionnement des services, l'allocation des crédits ou la nomination de fonctionnaires doit d'abord lui être soumise afin qu'il l'étudie et l'approuve le cas échéant, avant d'en recommander l'adoption au Conseil. Ce type d'organisation entraîne un fonctionnement

22 Son verdict est clair: «1. L'administration des affaires de la Cité de Montréal, par son Conseil depuis 1902, a été saturée de corruption provenant de la plaie du patronage; 2 . La majorité des échevins a administré les commissions et le conseil de manière à favoriser l'intérêt particulier de leurs parents et amis, auxquels on distribuait contrats et positions au détriment des intérêts généraux de la Cité et des contribuables; (...) 6. Tout le monde s'accorde à condamner ce système, qui a donné naissance au patronage et à ses abus». DGDA, Rapport de la Commission (1909), 73-74.

23 H. Parent, loc. cit., 363; R. Rumilly, op. cit., 404.

24 Francine Gagnon-Lacasse, Évolution des institutions politiques de la ville de Montréal (1921-1965), mémoire de M.A. (sciences politiques), Université de Montréal, 1967, 39-41, 122-126.

25 Les «hauts fonctionnaires» supérieurs, tels l'avocat en chef, le greffier et ses adjoints, les évaluateurs, le trésorier et autres demeurent responsables devant le Conseil municipal: Honoré Parent, loc. cit., 363-364; Francine Gagnon-Lacasse, op. cit., 124. 
d'une lourdeur sans précédent. Tous les membres du Bureau doivent ainsi se rencontrer quotidiennement, afin de régler les nombreux problèmes que rencontre l'administration d'une Ville de la taille de Montréal. Les chefs de service, quant à eux, ne peuvent prendre la moindre décision sans fournir des renseignements détaillés au Bureau des commissaires ${ }^{26}$. On imagine sans mal l'encombrement qui doit exister au Bureau des commissaires, car la municipalité compte en 1914 plus de 2000 fonctionnaires, cols blancs et cols bleus compris ${ }^{27}$. On ne sera donc pas surpris d'apprendre que les années de gouvernement réformiste se soldent par une augmentation considérable des dépenses d'administration ${ }^{28}$.

La question de l'organisation du travail et de la nomination des fonctionnaires refait de nouveau surface au cours du second mandat des réformistes. Le Bureau des commissaires étudie alors la possibilité de créer un «service municipal [...] dans le but d'établir différentes classes d'employés, leur salaire et le système de promotion basé sur leur mérite respectif ${ }^{29} \gg$. Mais après quelques mois de réflexion sur la question, le Bureau rejette le projet parce qu'il «ne croit pas judicieux de créer un bureau général [...] vu que des qualifications spéciales sont requises pour chaque département ${ }^{30}{ }_{\text {». Ainsi, même les réformistes }}$ alors au faîte de leur pouvoir reculent devant l'idée de soumettre les questions relatives aux fonctionnaires à une tierce institution, préférant dans ce cas comme dans tous les autres conserver la haute main sur les décisions.

Du côté de ses réalisations, le bilan du Bureau des commissaires n'est pas plus concluant. D'abord, les réformistes n'ont pas vraiment cherché à résoudre les problèmes de fond auxquels la Ville est alors confrontée. En dépit de sa prétention à répondre aux besoins de la Ville dans son ensemble, l'équipe au pouvoir traite l'électorat avec un élitisme qui la conduit à favoriser les quartiers de l'ouest de Montréal au détriment de ceux de l'est, comparativement moins bien nantis ${ }^{31}$. Voilà qui explique en gros pourquoi, en 1914, l'électorat montréalais

26 DGDA, Service municipal, Rapport du Bureau de recherches municipales de New York sur l'administration de Montréal (1917), 120, 274.

27 Chiffres compilés d'après les prévisions budgétaires pour l'année 1915: DGDA, dossier Budget-conseil.

28 Michael Gauvin, loc. cit., 21.

29 DGDA, Rapports et dossiers de résolution du Conseil municipal et du Comité exécutif (Rapports), $2^{c}$ série, $n^{\circ} 4418$; requête de l'échevin Marcil au Bureau des commissaires, 23 janvier 1911.

30 DGDA, idem, $\mathrm{n}^{\circ}$ 2428; rapport du Bureau des commissaires, 25 avril 1912. 89.

31 Francine Nagant, op. cit., 288. Voir également la thèse d'Annick Germain, op. cit., 
confie la mairie à Médéric Martin, manufacturier de cigares et député libéral fédéral, mettant ainsi fin à la seconde parenthèse réformiste ${ }^{32}$.

Les populistes héritent d'une situation financière difficile. En quatre ans, sous les réformistes, les dépenses d'administration ont en effet décuplé. Jusqu'en 1914, la hausse des dépenses s'est produite dans une période où le revenu municipal s'est accru considérablement, grâce à l'augmentation des valeurs immobilières. Telle n'est cependant plus la situation à partir de 1914 et les dépenses se maintiennent, voire continuent leur ascension, alors que les revenus stagnent ${ }^{33}$. Le problème était pourtant à prévoir et avait déjà été soulevé au moins par le trésorier, avant la période de hausse des valeurs immobilières enregistrée entre 1910 et 1913. Ce dernier, tout comme son successeur, n'avait pas manqué d'insister sur la nécessité d'augmenter les revenus de la Ville ${ }^{34}$. Mais la solution préconisée à cette fin, hausser le taux de la taxe foncière, est de celles que bien peu de politiciens osent adopter tant elle mécontente les électeurs. Par ailleurs la politique d'annexion des Villes environnantes, inaugurée à la fin du XIX $^{e}$ siècle et encore en vigueur au milieu des années 1910, alourdit aussi le fardeau financier de Montréal. En continuant d'accroître ainsi son territoire ${ }^{35}$, la Ville hérite du même souffle des dettes accumulées par les municipalités qu'elle incorpore. Coup sur coup, en 1916 et en 1917, son bilan financier est déficitaire ${ }^{36}$.

Cette situation délicate rend de plus en plus imminente la nécessité de réformer le mode d'administration de la Ville. Au cours de l'année 1916, une commission spéciale composée d'échevins est mandatée par le Conseil pour enquêter sur les services. Mais très tôt déroutée par l'énormité de la tâche, la commission s'avère incapable de trouver des solutions pour réduire de manière significative les dépenses de fonctionnement ${ }^{37}$. Voilà sans doute pourquoi le Conseil municipal suggère finalement de confier cette enquête à des «experts compétents ${ }^{38}$. Tout comme d'autres villes canadiennes, dépassées

32 Par la suite, il lui confiera pendant douze ans le mandat de diriger les affaires de la Ville et au total, Médéric Martin sera élu cinq fois maire de Montréal entre 1914 et 1928, à l'exception d'un mandat de deux ans entre 1924 et 1926; DGDA, Dossiers historiques n D-025, microfilm, bobine 84 .

33 DGDA, rapport annuel du trésorier de la Cité (1914), 4.

34 GDA, voir en particulier les rapports annuels du trésorier de 1914, 1915 et 1916.

35 Andrew Sancton, «Montreal», Warren Magnusson et Andrew Sancton, dir., City Politics in Canada (Toronto, University of Toronto Press, 1983), 65.

36 Honoré Parent, loc. cit., 365-366.

37 DGDA, Dossiers historiques $\mathrm{n}^{\circ}$ D001.2-6, microfilm, bobine 2; discours du Commissaire E. W. Villeneuve au Monument National, 2 novembre 1916, 8.

38 DGDA, rapport annuel du greffier 1916; résolution du Conseil municipal, 4 décembre 1916. 
elles aussi par la croissance rapide de leur administration, Montréal se tourne vers une firme d'experts américaine en vue de trouver des solutions aux nombreux problèmes de gestion auxquels elle est confronté $e^{39}$. Le 12 septembre 1917 elle engage donc le Bureau de recherches municipales de New York, en lui confiant le mandat de faire une étude complète sur son administration ${ }^{40}$.

Les recommandations des experts sont en tous points conformes aux visées du mouvement réformiste. Ainsi, le Bureau suggère de confier la direction de l'Hôtel de Ville à un petit conseil élu composé de sept à neuf membres qui, à son tour, choisirait un gérant municipal chargé de l'administration des affaires. Ce gérant, expliquent les experts, «assumerait le pouvoir exécutif qui fait défaut dans l'administration actuelle $e^{41}$ ». Ils retiennent l'idée de regrouper les services municipaux en quelques grands secteurs à la tête desquels seraient placés des chefs administratifs. Ces derniers, nommés par le gérant, auraient pour tâche de le seconder. Le Bureau insiste aussi fortement sur la nécessité de mettre sur pied une «commission du service civil», c'est-à-dire de la fonction publique, pour abolir le patronage (favoritisme). Dotée de tous les pouvoirs nécessaires, la nouvelle institution aurait pour fonction l'embauche des fonctionnaires, leur classification et la gestion de leurs promotions. Cette tentative des populistes pour améliorer la situation de la Ville ne semble pas jugée satisfaisante par tous ceux qui, gouvernement provincial en tête, souhaitent faire un grand ménage dans l'administration municipale. Et à peine le rapport déposé, le gouvernement de Lomer Gouin choisit d'imposer sa tutelle à Montréal.

\section{3 - LA COMMISSION ADMINISTRATIVE: UNE NOUVELLE FORME D'INCURSION DES RÉFORMISTES DANS L'ADMINISTRATION MUNICIPALE?}

Au mois de février 1918, le gouvernement provincial décide donc de nommer une Commission administrative dans le double but de remettre de l'ordre dans les finances de la Ville et d'en réduire les dépenses d'administration. La nouvelle institution doit entrer en

39 La ville de Toronto, notamment, fait aussi appel aux services du bureau new-yorkais en 1913: John C. Weaver, «The Modern City Realized: Toronto Civic Affairs, 1880-1915», Alan F. J. Artibise et Gilbert A. Stelter, eds., The Usable Past. Planning and Politics in the Modern Canadian City (Toronto, Macmillan, 1979), 58-59. En ce qui concerne les origines de ce type d'organisme et l'influence qu'il exerce dans le monde urbain des années 1910, voir l'ouvrage de Martin Schiesl, The Politics of Efficiency. Municipal Administration and Reform in America, 1800-1920 (University of California Press, 1977), 111-131.

40 DGDA, préambule du Rapport du Bureau de recherches..., op. cit., 9 février 1918.

41 Ibid., 132. 
fonction dès après les élections, prévues pour le début du mois d'avril $^{42}$. Bien que de courte durée, le règne de la Commission administrative sera déterminant quant à la forme que prendra l'administration municipale dans les années suivantes. Sont ainsi adoptées quantité de nouvelles mesures qui modifieront profondément les principes à la base de son mode de fonctionnement.

Nommée pour une durée initiale de quatre ans, la Commission se voit accorder de larges pouvoirs pour mener à bien son mandat. Le Conseil municipal et le maire sont maintenus dans leur fonction mais ils n'exercent plus qu'un rôle secondaire. La nouvelle instance détient pratiquement toutes les attributions dévolues autrefois au Conseil et au défunt Bureau des commissaires. En outre, parce qu'elle est nommée par le gouvernement provincial et non élue, la Commission a les coudées franches pour imposer tout un train de réformes, pour la plupart impopulaires. Dès les tout premiers jours qui suivent son entrée en fonction, elle donne le ton en imposant un régime d'austérité. Elle congédie d'abord les fonctionnaires en surnombre et augmente le pouvoir de taxation de la Ville. Les méthodes employées tranchent nettement avec l'ancien style de direction et choquent tout autant les fonctionnaires que la population ${ }^{43}$.

\section{La Commission du service municipal}

Pour introduire des réformes à plus long terme dans le fonctionnement de l'administration montréalaise, la Commission administrative s'inspire grandement du rapport d'enquête effectuée par le Bureau de recherches municipales de New York. À son tour, elle s'attaque à la délicate question de l'organisation du travail dans les services et tente de mettre sur pied une «commission du service civil». Parallèlement à la centralisation du pouvoir au sein d'un organisme exécutif, cette question est sans doute une des plus importantes pour les réformistes tant américains que canadiens. Instance intermédiaire entre les élus et les administrateurs, cette institution représente à leurs yeux la meilleure façon d'abolir le patronage à tous les niveaux, par l'instauration du principe de l'embauche et des nominations au mérite qu'elle induit, en lieu et place des pratiques de favoritisme qui dominent alors sur tous ces plans ${ }^{44}$.

42 La Presse, 30 janvier 1918, 1.

43 Les journaux montréalais font état quotidiennement des décisions prises par la Commission administrative au cours des premiers jours de son entrée en fonction. Ces articles donnent lieu à plus d'une critique à l'égard de la nouvelle équipe responsable de l'administration municipale. Voir notamment les éditions du quotidien La Patrie du 8 ainsi que du 12 avril 1918, 1, 3, respectivement. Voir aussi La Presse du 15 avril, 4, 7, de même que du 3 mai, 4.

44 Martin J. Schiesl, op. cit., 25-45. 
La Commission administrative doit cependant faire appel à une autre firme pour mener à bien le projet, en l'absence de directives proposées à ce chapitre par la firme de New York. Après un rapide sondage auprès des autres Villes canadiennes et américaines ${ }^{45}$, elle décide finalement d'engager la société Arthur Young, également au service du gouvernement fédéral au même moment ${ }^{46}$. À la fin de l'année 1919, les experts présentent le résultat de leur labeur à la Commission, contenu dans un rapport de plusieurs centaines de pages comportant la classification de tous les fonctionnaires et une description détaillée de chaque catégorie d'emploi ${ }^{47}$. La complexité de l'ensemble a de quoi surprendre. Les milliers d'employés que compte l'administration montréalaise sont répartis dans plus de 200 sousclasses distinctes ${ }^{48}$.

Dans la missive qu'il fait parvenir aux chefs de service au début de l'année 1920, le directeur de la nouvelle Commission du service municipal prend bien soin de préciser que le volumineux document n'est pas définitif et qu'il pourra être ajusté en cours de route ${ }^{49}$. Vraisemblablement, il tente ainsi d'endiguer l'émoi que ne manque pas de causer la classification. Pourtant, toutes ces réformes sont bien accueillies par plusieurs chefs de service qui les réclamaient depuis longtemps. Déjà en 1909, lors de la commission d'enquête Cannon sur l'administration municipale, certains avaient exprimé leurs doléances et réclamé un accroissement de leurs pouvoirs sur le fonctionnement interne de leurs départements respectifs. Mais la centralisation extrême du processus de décision qui résulte de l'avènement du Bureau des commissaires n'apporte pas d'amélioration à cet égard, bien au contraire. Dès lors, les chefs de service se butent à la lourdeur du fonctionnement qui se traduit par la lenteur avec laquelle sont prises les décisions, voire même par l'incapacité d'adopter les réfor-

45 DGDA, Rapports, $3^{\mathrm{e}}$ série, $\mathrm{n}^{\circ}$ 6894; Ernest Décary, président de la Commission administrative à René Bauset, greffier, 21 janvier 1919.

46 DGDA, Idem, $\mathrm{n}^{\circ}$ 7473; Arthur Young and Company, «Accountants and Auditors, Efficiency and Industrial Engineers», à la Commission administrative, 29 janvier 1919. Sur l'histoire de la Commission du service civil du gouvernement fédéral, voir l'ouvrage de J.E. Hodgetts et al., Histoire d' une institution. La Commission de la Fonction publique du Canada, 1908-1967 (Québec, Presses de l'Université Laval, 1975), 581 p. L'épisode qui a trait à l'enquête des experts américains à Ottawa est abordé dans les chapitres trois et quatre.

47 DGDA, Service municipal, Classification des fonctionnaires. Attributions et salaires, expertise par Arthur Young \& Co., 1920.

48 Pour chacune d'entre elles, la firme a établi une description détaillée des tâches, qui inclut une échelle de salaires et précise la nature des qualifications requises en terme d'expérience professionnelle et de formation; DGDA, ibid.

49 DGDA, Dossiers collectifs, 1920-1; missive du Commissaire du service municipal, $1^{\text {er }}$ janvier 1920. 
mes demandées pour améliorer l'organisation des services devenue vétuste ${ }^{50}$.

De leur côté, bien des fonctionnaires voient aussi d'un bon œil l'introduction de tous ces réaménagements, et la perspective de la classification des emplois semble même rassurante à plusieurs égards $^{51}$. Si la classification présente peut-être le désavantage de réduire les possibilités d'obtenir des privilèges, elle permet en revanche d'espérer pouvoir bénéficier de promotions ou d'augmentations de salaire en fonction de critères précis et fixes, et d'échapper ainsi à l'arbitraire avec lequel ils étaient jusqu'alors accordés.

Mais en dépit des appuis certains dont bénéficie la classification, l'avenir de cette entreprise ne sera pas pour autant assuré. Et le nouveau projet de réforme, encore une fois, va rejoindre tous les autres dans la voûte des archives, peu de temps après le départ de la Commission administrative. Quant à elle, la Commission du service municipal ne connaît pas un avenir plus prometteur alors que le Conseil municipal obtient tout bonnement l'abrogation de l'article de la charte qui s'y rapporte, en $1925^{52}$. Il faut attendre le début des années 1930, et le contexte de restrictions budgétaires entraîné par la crise économique, pour que soit reprise l'idée d'une classification des fonctionnaires.

Malgré cet échec à court terme, la Commission administrative n'en réussit pas moins à introduire, dans l'enceinte de l'Hôtel de Ville, les ferments propices à l'implantation d'un nouveau mode de gestion de l'appareil municipal. Après plus de vingt ans de déclarations d'intention et de projets de réforme à peine esquissés, elle parvient à mettre en place un cadre favorable à la formation d'une bureaucratie «moderne». Et même si plusieurs aspects de cette réforme seront graduellement minés au fil des années 1920 , la période qui s'amorce à l'aube de la crise favorisera la résurgence de tous les projets élaborés une décennie plus tôt.

\section{Vers l'adoption d'une nouvelle charte}

Dans l'immédiat, cependant, plusieurs tolèrent mal les méthodes de ces «messieurs les administrateurs», jugées radicales, voire dictatoriales. Les politiciens, au premier chef, voient d'un bien mauvais œil le déploiement de toutes ces mesures bureaucratiques qui visent à

50 Cette question est abordée en détail dans le chapitre trois de ma thèse, op. cit.

51 DGDA, Dossiers collectifs, 1919-1-01; allocution du président de l'Union mutuelle des employés civiques, s.d.

52 Statuts du Québec, $15 \mathrm{Geo} \mathrm{V}$, chap. 92, section 7, Loi modifiant la charte de la Cité de Montréal, sanctionnée le 3 avril 1925. 
resserrer le contrôle de leurs pratiques. Ils réclament haut et fort la restauration de l'autonomie de la Ville ${ }^{53}$. Face au mécontentement grandissant qui fuse de toutes parts, le gouvernement provincial doit retirer la Commission administrative un an avant la fin de son mandat prévue pour 1922. Au printemps 1921, la population montréalaise est de nouveau appelée à se prononcer sur le mode de gouvernement municipal. Elle opte pour le maintien de l'élection des échevins dans les quartiers et l'avènement d'un comité d'administration, chargé d'exercer le pouvoir exécutif ${ }^{54}$.

Avec l'introduction de la charte de $1921^{55}$, le principe de la séparation des pouvoirs est consolidé. L'arrivée dans le paysage politique montréalais d'un Comité exécutif, composé de cinq conseillers municipaux choisis au sein du Conseil, vise également à restreindre les attributions du maire et devient en somme le lieu véritable de l'exercice du pouvoir ${ }^{56}$. C'est désormais le Comité exécutif qui prépare le budget et qui, après l'avoir soumis pour approbation au Conseil municipal, gère les crédits alloués. Il se voit attribuer l'entière intitiative en matière réglementaire et toute décision doit recevoir son aval. À partir de 1922, il possède en outre seul le pouvoir de nomination et de contrôle de l'ensemble des fonctionnaires.

La charte de 1921 confirme l'importance des services municipaux et reconnaît ainsi l'existence d'une sphère administrative spécifique. Les services sont désormais divisés en six ensembles: les finances, le secrétariat, le contentieux, la santé, les travaux publics et la sécurité publique. Elle accorde aussi certains pouvoirs aux chefs qui les dirigent: ils peuvent nommer et révoquer leurs fonctionnaires, fixer les augmentations de salaires et les promotions, mais doivent soumettre le tout au Comité exécutif devant lequel ils sont responsables. Pour faciliter les communications entre ces différentes instances, une disposition de la charte prévoit la nomination d'un directeur des services responsable, pour sa part, devant le Conseil. Il assiste à toutes les réunions du Comité exécutif et constitue ainsi le principal moyen de contrôle de l'administration dont dispose le Conseil. Le poste de directeur des services est donc un poste-clé de l'administration municipale; au cours des années 1930, l'avocat Honoré Parent, qui l'occupe, lui fera prendre une importance encore plus grande que celle prévue dans la charte de 1921.

\footnotetext{
53 Robert Rumilly, op. cit., 8-11; Honoré Parent, loc. cit., 367.

54 Paul-André Linteau, op. cit., 412; Robert Rumilly, op. cit., 29-31.

55 Statuts du Québec, 11 George V, chapitres 111 et 112 (cédule B), Loi amendant la charte de la Cité de Montréal, 1921.

56 Voir à cet égard le mémoire de Francine Gagnon-Lacasse, op. cit., 160; de même que l'ouvrage de Paul-André Linteau, op. cit., 412-413.
} 
En définitive, cette phase initiale de la bureaucratisation municipale se caractérise par l'adoption de plusieurs réformes majeures. Cette étape marque ainsi l'avènement d'un réaménagement global de l'administration montréalaise, dont la séparation des pouvoirs, la reconnaissance de la sphère administrative et la réorganisation des services municipaux constituent les aspects les plus visibles. Mais ces changements au niveau formel ne sauraient suffire en eux-mêmes à modifier substantiellement le fonctionnement quotidien de l'administration. Bien que toutes les structures nécessaires à la «modernisation» de l'administration et à sa «rationalisation» soient adoptées, plusieurs indices laissent supposer que des pans entiers de son fonctionnement n'adoptent pas forcément le même tempo. Aux structures nouvelles se juxtaposent des pratiques anciennes, en particulier pour ce qui concerne les mesures reliées à la gestion du personnel: embauche, classification des emplois, attribution des promotions et des augmentations de salaires, normes disciplinaires; mesures, on le soupçonne aisément, qui peuvent s'avérer très rentables sur le plan politique.

\section{I - LA PÉRIODE 1930-1944: LA CONSOLIDATION PROGRESSIVE DES RÉFORMES}

C'est le populiste Camillien Houde qui domine le paysage politique montréalais au cours de cette seconde période, inscrite sous le sceau de la crise économique. Succédant à Médéric Martin ${ }^{57}$, Houde sera élu à la mairie durant quatre mandats, entre 1928 et 1940. Mais il semble bien que la coloration que ce maire donne à la vie politique municipale au cours de la période provient plus de son style marquant que de son emprise réelle sur l'administration ${ }^{58}$. Ce qui caractérise avant tout cette période, de notre point de vue, est l'importance croissante prise par la sphère administrative, par l'intermédiaire des chefs de service.

L'influence de ces acteurs n'est pas nouvelle et se manifeste très certainement déjà au tournant du siècle, alors que Montréal, tout

57 R. Rumilly, op. cit., 111-113.

58 Très engagé en même temps sur la scène politique provinciale, Houde ne consacre qu'une partie de son temps à la direction de Montréal. Sur sa carrière politique voir le livre de Robert Lévesque et Robert Migner, Camillien et les années 1920, suivi de Camillien au goulag. Cartographie du houdisme (Montréal, Éditions des Brûlés, 1978), 183 p. Voir aussi l'étude de Claude Dupont, Camillien Houde, politicien de carrière, mémoire de M.A. (histoire), Université d'Ottawa, 1972, 164 p., et en particulier le chapitre deux consacré à sa carrière municipale. 
comme l'ensemble des municipalités, fait de plus en plus appel à un personnel spécialisé pour gérer ses services ${ }^{59}$. Mais les nouvelles règles du jeu définies dans la charte municipale de 1921 et la situation de crise économique dans laquelle est plongée l'administration montréalaise au cours des années 1930, confèrent une visibilité plus grande aux chefs de service et leur permettent d'exercer une influence croissante sur les décisions.

\section{L'importance accrue des chefs de service}

L'importance grandissante de ces hauts fonctionnaires se vérifie nettement dans le cas du directeur des services puis dans celui des finances. L'avocat Honoré Parent est vraiment la figure centrale de l'administration montréalaise entre 1930 et 1944 . Il entre au service de la Ville en 1922, d'abord dans le département du contentieux ${ }^{60}$. Il est nommé directeur des services en 1930, au début du deuxième mandat de Camillien Houde à la mairie. Il devient ainsi le deuxième fonctionnaire à occuper ce poste depuis sa création en 1921. Par sa position d'intermédiaire entre les sphères politique, exécutive et administrative, il se situe au cœur de l'administration et toute information passe théoriquement et très certainement par lui, avant d'être acheminée vers son destinataire. Cet homme, proche du Parti libéral, semble bénéficier de l'estime sinon du respect de plusieurs, et surtout des hommes d'affaires et des financiers ${ }^{61}$. En mai 1940, au moment où Montréal est mise sous tutelle une seconde fois par le gouvernement provincial, Honoré Parent est choisi comme administrateur délégué de la Commission municipale de Québec ${ }^{62}$. Lui seul alors semble capable de faire appliquer les directives imposées par le gouvernement provincial ${ }^{63}$.

Louis Fabien Philie représente la seconde figure dominante de l'administration municipale au cours de la période. Engagé en 1929 à

59 Voir à cet égard l'ouvrage de Jon C. Teaford, op. cit. Dans le contexte montréalais, bien rares sont les études qui se sont penchées sur cette question. Une des recherches les mieux connues est certainement celle de Michael Farley, Othmar Keel et Camille Limoges qui porte sur le cas du service de santé de la municipalité: «Les commencements de l'administration montréalaise de la santé publique (1865-1885)», HSTC Bulletin. Journal of the History of Canadian Science, Technology and Medecine/Revue d' histoire des sciences, des techniques et de la médecine au Canada, 20 (1982): 24-46; 21 (1982): 85-109. Voir aussi la maîtrise en cours de Ginette Gagnon, Le projet de filtration de l'eau: Montréal 1890 a 1914, département d'histoire, Université de Montréal.

60 DGDA, dossier personnel d'Honoré Parent.

61 Robert Rumilly, op. cit., 244; aussi tome 5: (1939-1967) de son Histoire de Montréal (Montréal, Fides, 1974), 14.

62 DGDA, dossier personnel d'Honoré Parent; extrait du procès-verbal de la Commission municipale de Québec, 17 mai 1940.

63 Robert Rumilly, op. cit., tome 5: 18. 

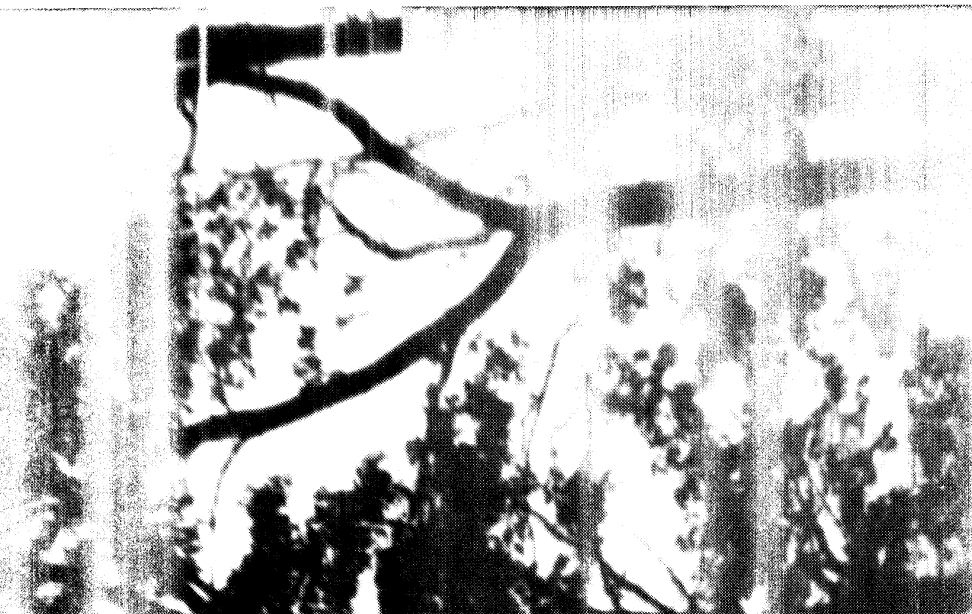

- 1 r pus

ale $-1,0$

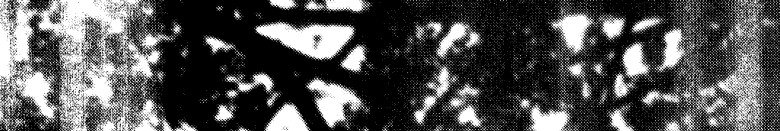

1.1. $50.6 x+2$ 1) $-x+y$ - 1370

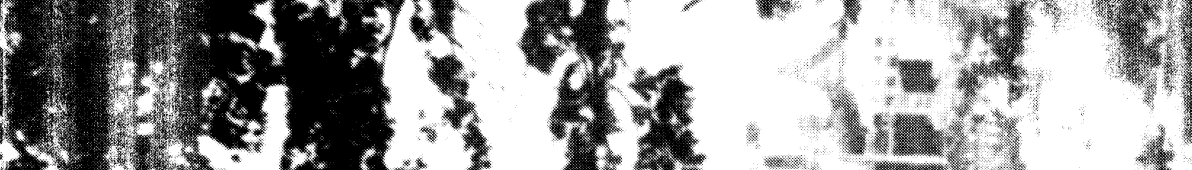

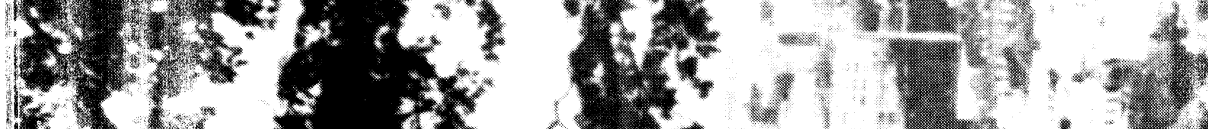

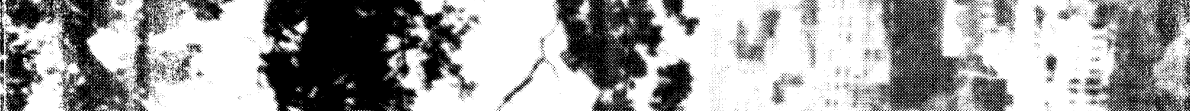
(1)

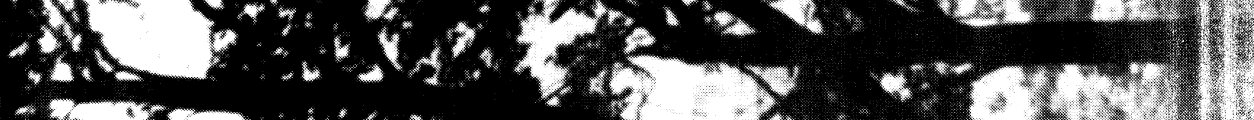

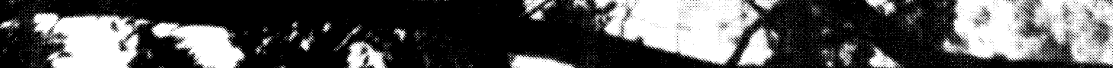

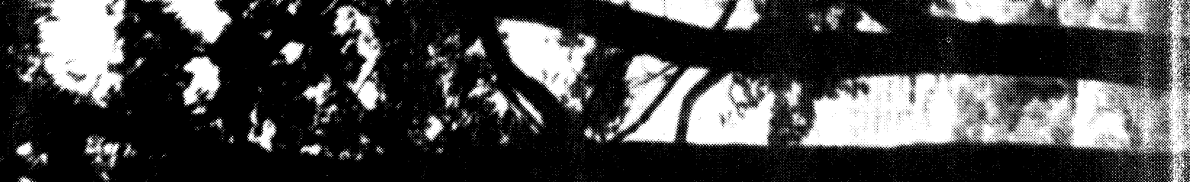

(Novers.

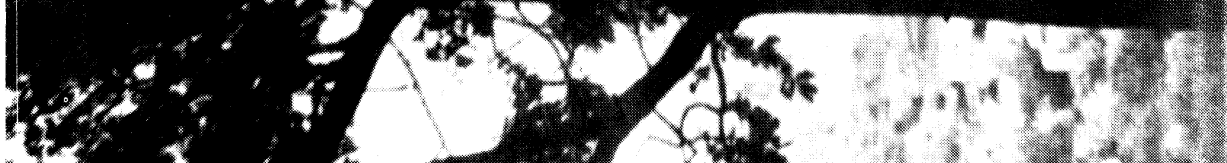
1.

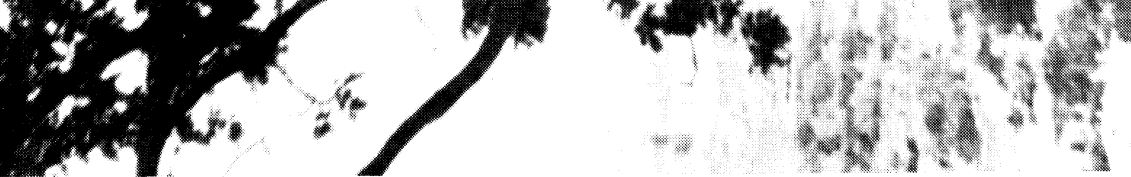


titre de trésorier de la cité, il est un des tout premiers hauts fonctionnaires à occuper un poste de direction sans avoir eu à gravir les échelons de la hiérachie interne pour y parvenir. Jusque-là, il était assistant-gérant général de la Banque provinciale ${ }^{64}$. Dès son entrée en fonction, il effectue une première réorganisation de certaines sousdivisions placées sous sa direction ${ }^{65}$. Mais c'est surtout par le rôle de premier plan qu'il joue au cours d'une enquête menée dans son service entre 1932 et 1936 qu'il s'illustre. Fervent partisan de la modernisation des méthodes employées dans son service, il contribue activement à la réorganisation du secteur et à sa centralisation.

\section{Difficultés financières et favoritisme}

Au cours des années 1930, deux questions dominent la vie politique locale: les problèmes économiques et sociaux et la gestion des services municipaux, jugée inadéquate par plusieurs contemporains. La période révèle tout d'abord l'ampleur des difficultés financières auxquelles l'administration montréalaise est plus que jamais confrontée, alors qu'aux anciens problèmes hérités des décennies précédentes, se greffe le fardeau de la crise économique. Il faut dire que Montréal, tout comme les autres Villes canadiennes, assume une grande partie des services sociaux existants. Et comme la population montréalaise est particulièrement affectée par la récession, les responsabilités sociales de l'administration municipale montent en flèche ${ }^{66}$.

La crise qui secoue la Ville ébranle aussi la confiance de beaucoup vis-à-vis de l'administration dirigée depuis 1928 par Camillien Houde. En 1932, des scandales éclatent autour de l'attribution des secours directs ${ }^{67}$. Le problème du favoritisme à l'embauche ou lors des promotions prend une nouvelle ampleur. Si cette pratique est

64 DGDA, dossier personnel de Louis Fabien Philie.

65 DGDA, Rapports, $3^{e}$ série, $\mathrm{n}^{\circ} 36498$; mémoire soumis au directeur des services, 24 février 1930.

66 En 1933 par exemple, Montréal distribue de l'aide à 30\% de la population globale; Paul-André Linteau, op. cit., 379. Pour faire face à ce développement important de ses dépenses, la Ville a recours à de nouveaux impôts à partir de 1935: une taxe de vente de $2 \%$ et un impôt sur le revenu. Mais ces sources supplémentaires de revenu s'avèrent rapidement insuffisantes et n'empêchent pas l'accroissement rapide de la dette; Raymond Tanghe, Montréal (Montréal, Éd. Albert Lévesque, 1936), 166-167.

67 On s'insurge particulièrement contre le fait que l'administration municipale a choisi les secrétaires des conseillers municipaux, alors houdistes en grande majorité, comme agents pour distribuer ces secours; Claude Dupont, op. cit., 69-70; Terry Copp, «Montreal's Municipal Government and the Crisis of the 1930s», Alan F. J. Artibise et Gilbert A. Stelter, eds., The Usable Past. Planning and Politics in the Modern Canadian City (Toronto, Macmillan, 1979), 119. 
certainement plus visible et importante dans des services tels les travaux publics ou celui de la police, elle n'est pas pour autant absente des autres départements qui regroupent une majorité de cols blancs. Dans le contexte de raréfaction des emplois, les pressions sur l'administration municipale en vue d'embaucher les nombreux chômeurs affluent de toutes parts: de la population dans son ensemble mais aussi des conseillers municipaux en poste dans les différents quartiers. À la multitude de lettres de demandes d'emploi que reçoivent le Comité exécutif et les chefs de service, il n'est pas rare de trouver accolées les recommandations empressées des conseillers ${ }^{68}$.

Si cette façon de procéder irrite fort probablement certains chefs de service de même que les réformistes bien pensants, elle n'en constitue pas moins une pratique courante à l'époque avec laquelle tous sont forcés de composer. Au-delà du jugement négatif que suscitent ces pratiques, il importe d'y réfléchir pour tenter, entre autres, de dégager leur signification pour les personnes qui y ont recours à cette époque, et aussi pour comprendre pourquoi elles se perpétuent avec autant de vigueur en dépit des nombreuses tentatives adoptées en vue de les faire cesser.

Comme on l'a démontré plus haut, cette question est intimement liée à celle de l'exercice du pouvoir. Le maintien de ces pratiques, particulièrement dans l'embauche, s'explique par la volonté du Comité exécutif et plus globalement des élus de conserver leur droit de regard sur la sphère administrative qui tend de plus en plus à leur échapper, à mesure que l'administration municipale se bureaucratise. En outre, dans le contexte des années 1930, les conseillers tout autant que le maire sont soumis à d'intenses pressions de la part de la population qui tente d'obtenir de l'emploi et d'échapper ainsi à la pauvreté due au chômage ${ }^{69}$. Sans vouloir nier le capital politique qu'ils cherchent à accumuler en tentant à leur tour d'obtenir gain de cause au

68 Cette requête parmi d'autres d'Hector Dupuis, échevin du quartier Papineau et membre du Comité exécutif, est éloquente: «Comme le département de la carte d'identité doit fonctionner prochainement, j'ose espérer que le quartier Papineau aura sa quote-part des inspecteurs qui seront engagés. La présente est pour vous introduire monsieur B.... rue De Montigny Est qui, jusqu'à maintenant s'est spécialisé dans des enquêtes de toutes sortes; je le crois donc absolument compétent pour remplir la charge d'enquêteur... Voudriez-vous donc, si mon quartier est appelé à fournir de ces enquêteurs, considérer cette recommandation de ma part comme la première à laquelle vous devrez apporter votre bonne attention»: DGDA, Rapports, $3^{\mathrm{e}}$ série, $\mathrm{n}^{\circ}$ 67743-1; Hector Dupuis, conseiller, à J. E. Gauthier, greffier de la cité, 8 juin 1934.

69 C'est bien souvent par dizaines que les conseillers ou le maire reçoivent quotidiennement des demandes d'embauche et d'aide de toutes sortes des électeurs des quartiers qu'ils représentent. Voir le témoignage d'un conseiller municipal alors qu'il dénonce ces pratiques; J. A. Edmison, "The Curse of Civic Patronage», Municipal Review of Canada, 35,7 (juillet 1939): 11-12. 
nom de leurs électeurs auprès de l'administration ${ }^{70}$, la prise en compte de leur position dans ce contexte permet de mieux comprendre leurs nombreuses interventions.

Il faut voir aussi que les fonctionnaires qui sont embauchés selon ces procédés ne sont pas forcément incompétents, contrairement à l'opinion généralement répandue ${ }^{71}$. Davantage qu'une façon de se soustraire à des examens d'entrée ou à un processus de sélection quelconque, le recours à un conseiller municipal constitue sûrement pour la majorité le moyen le plus connu ou le plus accessible de faire parvenir une demande d'emploi à l'Hôtel de Ville, perçu déjà à cette époque comme une immense bureaucratie composée de milliers d'individus et dont les règles de fonctionnement échappent à plusieurs $^{72}$.

Ce type de rapports est aussi intimement lié à la question de la redistribution politique des ressources entre les gouvernants et les gouvernés. Dans le contexte de la modernisation de l'administration, et celui plus spécifique de la crise économique, la façon dont ces ressources doivent être redistribuées devient un enjeu fondamental. S'opposent alors deux conceptions dont l'une, plus ancienne certains diraient rétrograde - , est apparentée au patronage et l'autre, à la bureaucratie. Comme l'explique le politicologue Vincent Lemieux:

[...] les individus et les groupes faisant partie des publics administrés se sentent menacés par la bureaucratisation de la redistribution politique. Quelques-uns, bien sûr, craignent la perte de leurs privilèges, mais la menace la plus généralement ressentie est plutôt celle de l'impersonnalisation, de l'anonymat qui caractérise le rapport administratif quand il devient bureaucratique. Dans cette perspective, le patronage, que nous opposons

70 Il est bien connu que l'exercice du favoritisme constitue en un sens une forme d'échange en vertu de laquelle la personne qui obtient une faveur de son conseiller s'engage à lui donner son vote, à travailler pour sa campagne électorale, etc. Robert Lévesque et Robert Migner dans leur ouvrage sur Camillien Houde relatent le fait que, selon les dires de contemporains qu'ils ne nomment pas, les fonctionnaires pouvaient payer $200 \$$ et même plus à leur échevin, pour obtenir un emploi à l'Hôtel de Ville; Camillien et les années vingt..., op. cit., 114-115.

71 L'analyse des dossiers individuels des fonctionnaires permet de constater que bien des candidats qui font appel à leur conseiller ou au maire pour obtenir un poste possèdent souvent une expérience préalable dans le secteur du travail de bureau de même qu'une formation professionnelle.

72 Cette façon de procéder n'est pas unique à Montréal. Cindy Sondik Aron, qui a mené une étude sur les fonctionnaires de l'administration fédérale américaine, relève la présence de cas analogues: Ladies and Gentlemen of the Civil Service. Middle-Class Workers in Victorian America (New York, Oxford University Press, 1987), chapitre cinq. 
ici à la bureaucratie, se caractérise bien plus par la personnalisation que par la corruption d'un rapport social ${ }^{73}$.

Cette tension que Vincent Lemieux identifie lorsqu'il oppose le patronage à la bureaucratie prend tout son sens dans le cas des transformations qui se manifestent au sein de l'administration municipale, au cours des premières décennies du $\mathrm{XX}^{\mathrm{e}}$ siècle.

\section{La réorganisation du service des finances}

Financiers et banquiers exercent eux aussi de multiples pressions à l'endroit de l'administration et exigent qu'elle réforme son mode de gestion des finances, dans le but d'en faciliter l'examen. Lors des élections d'avril 1932, Fernand Rinfret, qui a mené sa campagne sur cette question de même que sur le problème du favoritisme, remporte la victoire contre Houde ${ }^{74}$. Dans la foulée, la nouvelle administration décide d'entreprendre une enquête en profondeur sur l'organisation des finances ${ }^{75}$.

Elle confie la tâche de revoir la comptabilité de la Ville aux maisons de vérificateurs comptables P. S. Ross \& Sons et Larue \& Trudel, en bons termes, dit-on à l'époque, avec le gouvernement libéral de Taschereau ${ }^{76}$. Les comptables, qui commencent leur travail d'enquête en mai 1932, remettent un premier rapport de 250 pages, avant la fin de l'année ${ }^{77}$. La plupart de leurs recommandations vont être adoptées dans les mois suivants ${ }^{78}$. Elles visent à favoriser une meilleure centralisation et supervision des coûts reliés à l'administration, et en particulier de ceux effectués par les chefs de département, par le contrôle plus étroit des dépenses de même que l'établissement de prévisions budgétaires plus précises. Le directeur des finances, nouvelle fonction créée à la suggestion des enquêteurs, se voit confier des attributions plus importantes que celles détenues jusque-là par le trésorier, son prédécesseur. Il cumule dès lors les

73 Vincent Lemieux, «Patronage ou bureaucratie», texte en appendice de son ouvrage Parenté et politique. L'organisation sociale dans l'Ile d'Orléans (Québec, Presses de l'Université Laval, 1971), 226.

74 Robert Rumilly, op. cit., 179; Terry Copp, loc. cit., 117-118.

75 DGDA, Rapports, $3^{\mathrm{e}}$ série, $\mathrm{n}^{\circ}$ 43834-1; extrait du procès-verbal, 22 avril 1932.

76 DGDA, Dossiers historiques $\mathrm{n}^{\circ}$ D400.1, microfilm, bobine 117; L'Illustration nouvelle, 27 octobre 1936. La maison P. S. Ross signe parfois des articles dans la Municipal Review of Canada (The Official Organ of the Union of Canadian Municipalities); voir notamment «The Value of Municipal Auditing», 21,3 (mars 1925).

77 DGDA, État financier de Montréal, rapport sur enquête par Ross \& Sons et Larue \& Trudel, 1932. 1933.

78 DGDA, Règlement 1230 concernant le directeur des finances et l'auditeur, 8 mai 
tâches de ce dernier et celles de contrôleur, en devenant responsable à la fois des finances et de la comptabilité de la Ville.

En septembre 1936, au moment où la Ville met fin à l'engagement des comptables, le mode de fonctionnement du département des finances est profondément transformé. Mais tout comme celles qui l'ont précédée en d'autres moments et à d'autres endroits de l'administration, cette nouvelle réforme cause bien des remous ${ }^{79}$. Les sources de mécontentement sont particulièrement vives chez les fonctionnaires du service des finances: certains ont été bousculés, voire rétrogradés, et d'autres, plus nombreux encore, acceptent mal l'introduction des machines comptables avec lesquelles ils doivent désormais travailler ${ }^{80}$.

\section{2 - LA SECONDE MISE EN TUTELLE DE L'ADMINISTRATION MONTRÉALAISE}

En dépit de la réorganisation complète du service des finances et des maigres économies générées par la réintroduction de la classification des emplois par le directeur des services à partir de $1933^{81}$, la situation financière s'aggrave à la fin des années 1930. C'est que Montréal, comme l'ensemble des autres Villes canadiennes, ne peut faire face aux responsabilités trop lourdes que la crise l'oblige à assumer $^{82}$. Les milieux financiers de Montréal, quant à eux, attribuent la situation délicate de la Ville à sa gestion inadéquate. Les associations d'hommes d'affaires soutiennent que ses difficultés sont dues au régime de corruption qui sévit à l'Hôtel de Ville et prétendent que l'aide sociale ne représente que $5 \%$ de la dette municipale. Même si on ne peut parler d'une résurgence des mouvements réformistes à cette époque, les pressions se font de plus en plus nombreuses en vue de modifier la constitution de la Ville ${ }^{83}$.

\section{La Commission municipale de Québec}

En 1940, rien ne va plus du côté des finances de la Ville, alors que les déficits budgétaires s'accumulent et que les banques refusent

79 Les journaux montréalais suivent avec assiduité les réformes adoptées au service des finances: DGDA, Dossiers historiques $n^{\circ} 400.1$, microfilm, bobine 117; La Presse, 16 septembre 1936, L'Illustration, 17 et 18 septembre 1936, Le Canada, 10 octobre 1936, notamment. Voir aussi le dossier personnel de Louis Fabien Philie; «On forme une cabale contre M. Philie à l'hôtel-de-ville», Le Canada, 11 oxtobre 1934.

80 DGDA, Rapports, $3^{\text {e }}$ série, $n^{\circ}$ 43834-6; mémoire soumis au Comité exécutif, 28 septembre 1936.

81 AVM, Idem, n 43834-6; Honoré Parent au Comité exécutif, 2 novembre 1936.

82 Alfred John Pick, The Administration of Paris and Montreal: a Comparative Study (Montréal, Witness, 1940), 100-101.

83 Francine Gagnon-Lacasse, op. cit., 173. 
d'accorder des prêts ${ }^{84}$. Nonobstant des pratiques parfois douteuses, il demeure que la principale cause du marasme financier, ce sont les dépenses provoquées par la crise, ce que confirmera d'ailleurs le rapport de la Commission d'enquête Rowell-Sirois sur les relations entre le Dominion et les provinces ${ }^{85}$. En accord avec les financiers montréalais, le gouvernement provincial décide malgré tout d'imposer une seconde tutelle à l'administration municipale et, à partir du mois de mai 1940, tous les pouvoirs de gestion sont confiés à la Commission municipale de Québec ${ }^{86}$. Au cours des quatre années suivantes, elle s'appliquera à redresser la situation financière de la Ville et sa réputation vis-à-vis des institutions bancaires, mais cela se fera au détriment des principes démocratiques, une fois de plus ${ }^{87}$.

La Commission détient dès lors tous les pouvoirs reliés à l'administration de la Ville. Concrètement, cela signifie que toute résolution et toute décision prises par quelque instance que ce soit doivent d'abord lui être soumises avant d'entrer en vigueur. Elle approuve aussi tous les budgets établis au cours de son mandat et possède le pouvoir exclusif de régler l'ensemble des questions qui se rapportent à la gestion du personnel. Pour la seconder, la Commission municipale ne choisit nul autre qu'Honoré Parent et le nomme administrateur délégué ${ }^{88}$. L'homme-orchestre de l'administration montréalaise se voit ainsi confier la délicate tâche de faire appliquer les décisions prises par la Commission, dont la direction est assurée par le président L. E. Potvin.

\section{La riposte des fonctionnaires}

Au même moment, le regain de l'activité syndicale qui se produit à travers tout le pays n'épargne pas l'administration montréalaise. Tout comme leurs collègues des autres municipalités, les fonctionnaires montréalais vont emboîter le pas aux mouvements de revendi-

84 Honoré Parent, loc. cit., 372.

85 Terry Copp, loc. cit., 113.

86 Ibid., 125.

87 Dès 1940, la Commission impose une nouvelle constitution à la Ville. Les amendements votés concernent alors essentiellement le Conseil municipal, sa composition et son mode de fonctionnement, mais ne modifient pas les autres institutions municipales. Honoré Parent, loc. cit., 373. Voir aussi Harold Kaplan, op. cit., 338-339.

88 À cette occasion, il lance un appel solennel à tous les chefs de service en les conviant à collaborer au redressement de la situation financière de la Ville: «Je compte sur chacun des chefs pour remettre la municipalité à flot. (...) Pour réussir, il faut d'abord assurer l'ordre et la discipline, et faire régner la plus stricte économie dans l'administration. (...) Que chacun, dès maintenant, prenne ses responsabilités, comme je le fais moi-même. C'est ce que le public et le Conseil attendent de nous.» DGDA, Dossiers collectifs, 1940 A-01; Honoré Parent aux chefs de service, 25 mai 1940. 
cation ouvrière qui se développent au cours de la Deuxième Guerre mondiale ${ }^{89}$. Cols blancs et cols bleus profitent ainsi de cette vague pour faire connaître leurs doléances, longtemps contenues, aux dirigeants de l'administration municipale. Si au cours de la crise les employés municipaux étaient dans une position relativement privilégiée par rapport aux travailleurs plus directement soumis aux aléas de la conjoncture économique, leur situation s'est rapidement dégradée depuis ${ }^{90}$. Les cols blancs, en particulier, cherchent à faire valoir leur point de vue auprès de l'administration en présentant un mémoire en juillet $1942^{91}$. Outre l'augmentation générale de leurs salaires, ils réclament la nomination d'une «Commission du service civil». Ils suggèrent aussi d'étudier l'introduction d'une convention collective pour régler leurs conditions de travail. En guise de réponse, ils ne reçoivent qu'un accusé de réception.

Le ton se durcit au cours de l'année 1943. Tout comme les cols bleus, les pompiers et les policiers, avant eux, les cols blancs demandent la reconnaissance de leur syndicat fondé en 1920. La Commission municipale, qui semble d'accord pour faire certaines concessions, refuse toutefois l'accréditation syndicale, alléguant le fait que les cols blancs sont affiliés à une centrale. Le 21 décembre 1943, les fonctionnaires municipaux ripostent en déclenchant la première grève de leur histoire. Ils décident finalement de retourner au travail le 13 janvier 1944, après avoir accepté de se soumettre à la procédure d'arbitrage obligatoire pour régler leurs griefs, en vertu de la «Loi des différends entre les services publics et leurs salariés», adoptée à la même époque ${ }^{92}$. À l'issue de plusieurs mois de négociation, une première convention collective est signée par les deux parties au début de juillet $1944^{93}$, qui définit en termes complètement nouveaux les rapports entre les fonctionnaires et la direction.

89 Sur la question du syndicalisme parmi les fonctionnaires municipaux à cette époque, voir l'étude de S. J. Frankel et R. C. Pratt, Municipal Labour Relations in Canada. A Study of Some Problems Arising from Coliective Bargaining Between Municipalities and Municipal Trade Unions (Montreal, The Canadian Federation of Mayors and Municipalities/The Industrial Relations Center, McGill University, 1954).

90 Malgré les indemnités de vie chère accordées par la Commission municipale, la forte inflation qui prévaut durant le conflit mondial rend leur situation précaire. DGDA, dossier personnel d'un fonctionnaire; Lactance Roberge, directeur des Finances, à Honoré Parent, administrateur délégué de la Commission municipale de Québec, 2 octobre 1942.

91 DGDA, Dossiers collectifs, 1942 1-1; mémoire soumis à Honoré Parent par le Syndicat catholique national des fonctionnaires municipaux, 7 juillet 1942. Voir aussi Jacques Lesage, Le syndicalisme chez les fonctionnaires municipaux de la ville de Montréal, mémoire de M.A. (relations industrielles), Université de Montréal, 1957, 10.

92 Au sujet de cette loi, voir l'ouvrage de Jacques Rouillard, Histoire du syndicalisme au Québec, des origines a nos jours (Montréal, Boréal, 1989), 252.

93 DGDA, Dossiers collectifs, 1944 1-03; Convention collective intervenue entre la Ville de Montréal et le Syndicat des Fonctionnaires municipaux de Montréal, 6 juillet 1944. 
La syndicalisation des fonctionnaires, puis la signature de cette première convention collective s'inscrivent de plain-pied dans la période de consolidation des réformes, qui se déroule entre 1930 et 1944. Après la réorganisation du service des finances et l'adoption permanente du principe de la classification des emplois notamment, ces événements constituent une étape supplémentaire dans le processus de bureaucratisation qui s'élabore depuis le tournant du siècle. Si cette fois l'impulsion première provient des fonctionnaires, elle n'en participe pas moins de la même dynamique. Ces transformations constituent à leur manière une étape essentielle dans ce processus qui conduit à la réglementation toujours plus grande des multiples dimensions qui composent l'administration municipale. C'est maintenant au tour des fonctionnaires de se donner les outils nécessaires qui leur permettront dès lors de faire valoir leur point de vue. Face à l'institution complexe qu'est devenue l'administration municipale, ils n'avaient sans doute d'autre choix que de se regrouper autour d'un syndicat et de se définir, aussi de leur côté, un ensemble de règles qui leur assurent une représentation auprès des instances supérieures.

À la fin de l'année 1944, le visage de l'administration est complètement transformé. On est maintenant en présence d'une institution bureaucratisée, «moderne», à tout le moins au niveau formel. C'est que les moindres aspects de son organisation sont désormais consignés dans un ensemble étendu de règles écrites et fixes, en tous points conforme à l'idéal bureaucratique défini par Max Weber ${ }^{94}$ quelques décennies auparavant. On peut se demander si ces nouvelles conditions suffiront à assurer l'efficacité tant recherchée, à estomper les difficultés et les multiples contradictions que pose le fonctionnement quotidien d'une telle institution. Il est permis d'en douter. Mais il s'agit là d'une autre histoire...

\section{CONCLUSION}

C'est en vain qu'on chercherait à discerner dans les régimes successifs qu'a subis Montréal, une évolution constante, logique... (...)

L'histoire politique de cette Ville n'est qu'une suite de progressions suivies de reculs, d'arrêts, de recommencements voués à de nouveaux échecs. (...)

La Ville retombe toujours dans l'ornière des insuccès, des récriminations, des difficultés financières, en dépit de certains

94 Op. cit.; voir en particulier les pages 219 à 252. 
essais qui paraissaient heureux, et de certaines périodes de calme qui s'annonçaient durables ${ }^{95}$.

À l'issue de ce survol de l'histoire de l'administration municipale au cours de la première moitié du $\mathrm{XX}^{\mathrm{e}}$ siècle, nous serions tentée de faire nôtres ces paroles empruntées à Honoré Parent. Ce serait là supposer que le développement de cette institution ou de toute organisation quelle qu'elle soit puisse se faire selon un schéma prétabli, au gré d'une volonté ou suivant une logique unique. La période comprise entre le tournant du siècle et la fin de la Deuxième Guerre mondiale, étape particulièrement mouvementée de l'histoire de l'administration municipale, avec ses deux mises sous tutelle et les modifications considérables apportées à ses institutions, est là pour nous rappeler l'utopie d'une telle vision des choses. En effet, la transformation de l'administration montréalaise se produit d'une manière saccadée, caractérisée par une multitude d'avancées et de reculs. La reconstitution de cet épisode à partir de la scène administrative et non seulement politique permet de mettre au jour les luttes et les enjeux que les réformes introduites soulèvent.

Une première lecture des événements révèle l'existence de ces luttes sur la scène politique: luttes dominées par l'opposition entre réformistes et populistes. Alors que les premiers, qui prétendent vouloir dépolitiser l'administration, semblent favorables à sa bureaucratisation, les seconds s'y opposent dans la mesure où le faible développement de la sphère administrative leur permet de conserver leur emprise sur de multiples secteurs de la vie municipale. Mais une seconde lecture plus attentive démontre que le clivage entre les deux positions relève surtout de la rhétorique électorale et que ce sont d'abord des intérêts politiques qui sont en cause. Plus qu'à la volonté d'assainir l'administration, le désir de contrôler le cours pris par son expansion répond à des visées partisanes. C'est ce que nous révèle le mandat des réformistes entre 1910 et 1914, alors que ces derniers reculent devant l'adoption de plusieurs changements pour conserver la haute main sur l'administration. C'est aussi ce qui se dégage de l'enquête de 1917 sur l'administration, enquête commandée par le populiste Médéric Martin. Face aux nombreux problèmes qui secouent l'administration et à la menace de tutelle qui se précise, le maire est prêt à entreprendre une réorganisation importante de l'appareil administratif municipal.

Cette deuxième lecture permet surtout d'éclairer l'existence d'un autre terrain de luttes qui opposent cette fois les hommes politiques

95 Honoré Parent, loc. cit., 327-328. 
aux administrateurs, aux bureaucrates. Ici, les enjeux sont différents. Il s'agit pour les seconds d'obtenir la reconnaissance de la spécificité de leur sphère d'activité et de l'importance de leurs fonctions. Ce qui sera partiellement réalisé avant 1930. Néanmoins, c'est au cours de la période de la crise et du début des années 1940 que se produit le véritable tournant. La position financière difficile dans laquelle se trouve la Ville constitue un puissant incitatif à reprendre les réformes; réformes qui permettent de réduire partiellement les dépenses de fonctionnement. Mais au-delà des soubressauts de la scène politique, la période marque la montée de hauts fonctionnaires nettement favorables à l'adoption de réformes. Ce sont d'abord eux qui assurent à moyen terme le succès des changements qu'ils s'emploient à promouvoir. Quoique pour des motifs bien différents, une majorité de fonctionnaires participent aussi à cette dynamique de changement. Par leur désir de redéfinir les rapports de travail dans ce nouveau contexte, ils contribuent aussi à leur façon au processus de bureaucratisation amorcé au début du siècle.

Au moment où l'on interroge ces immenses bureaucraties que sont devenues les administrations publiques, il nous a semblé intéressant de nous pencher sur les conditions à l'origine de l'émergence mais aussi de la consolidation de la bureaucratie municipale montréalaise. 